Case Report

\title{
A Rare Case of Esophageal Dysphagia in Children: Aberrant Right Subclavian Artery
}

\author{
Claudia Barone, Nicolina Stefania Carucci, and Claudio Romano \\ Pediatric Department, University of Messina, Italy \\ Correspondence should be addressed to Claudio Romano; romanoc@unime.it
}

Received 11 November 2015; Revised 24 December 2015; Accepted 4 January 2016

Academic Editor: Bernhard Resch

Copyright (C) 2016 Claudia Barone et al. This is an open access article distributed under the Creative Commons Attribution License, which permits unrestricted use, distribution, and reproduction in any medium, provided the original work is properly cited.

\begin{abstract}
Dysphagia is an impairment of swallowing that may involve any structures from the mouth to the stomach. Esophageal dysphagia presents with the sensation of food sticking, pain with swallowing, substernal pressure, or chronic heartburn. There are many causes of esophageal dysphagia, such as motility disorders and mechanical and inflammatory diseases. Infrequently dysphagia arises from extrinsic compression of the esophagus from any vascular anomaly of the aortic arch. The most common embryologic abnormality of the aortic arch is aberrant right subclavian artery, clinically known as arteria lusoria. This abnormality is usually silent. Here, we report a case of six-year-old child presenting to us with a history of progressive dysphagia without respiratory symptoms. A barium esophagogram showed an increase of the physiological esophageal narrowing at the level of aortic arch, while at esophagogastroduodenoscopy there was an extrinsic pulsatile compression of the posterior portion of the esophagus suggesting an extrinsic compression by an aberrant vessel. Angio-CT (computed tomography) scan confirmed the presence of an aberrant right subclavian artery.
\end{abstract}

\section{Introduction}

Swallowing is an important function in maintaining optimal nutritional status [1]. It occurs in three phases: oral, pharyngeal, and esophageal. Dysphagia is any disturbance in swallowing, often described by the patients as a "perception" that there is an impediment to the normal passage of the swallowed material [2]. Oropharyngeal dysphagia involves the initial two phases and is rather common in patients with neurological impairment; esophageal dysphagia occurs at the third phase and is most commonly due to functional causes or anatomic abnormalities affecting the esophagus $[3,4]$. Early detection of dysphagia in infants and children is important to prevent or minimize complications because if not diagnosed this medical condition can lead to failure to thrive, aspiration pneumonia, gastroesophageal reflux, and/or the inability to establish and maintain proper nutrition and hydration [5]. A variety of medical conditions cause swallowing disorders in pediatric patients [6]. Aberrant right subclavian artery (ARSA) is a rare cause of dysphagia, but it must be taken into account in the differential diagnosis [7]. The majority of patients with this abnormality remain asymptomatic. In other cases ARSA may cause respiratory symptoms in children and dysphagia, cough, and chest pain in adults [7-10]. We report a rare case of six-year-old child presenting to us with a history of progressive dysphagia.

\section{Case Report}

A six-year-old child presented to our hospital with a progressive history of dysphagia, chest pain, and slow feeding. He did not have respiratory symptoms. His examination was normal with weight and length at the 90th percentile for age. His blood tests were normal. There were no abnormalities on ECG or echocardiography. A barium esophagogram showed an increase of the physiological esophageal narrowing at the level of aortic arch, being suggestive of extrinsic compression. At esophagogastroduodenoscopy, the esophageal mucosa was normal in appearance; biopsy examinations confirmed normal mucosa. About $15 \mathrm{~cm}$ from the buccal rhyme there was an extrinsic compression of the posterior portion of the esophagus that was pulsatile, suggestive of an aberrant vessel. Angio-CT (computed tomography) scan confirmed an aberrant right subclavian artery compressing the posterior middle third of the thoracic esophagus (Figure 1). This 


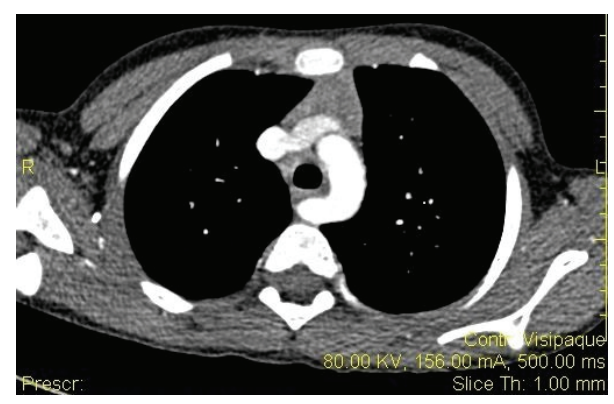

FIGURE 1: Angio-computed tomography: demonstrating the aberrant right subclavian artery compressing the esophagus.

artery originated from an aneurismal dilatation $(11 \mathrm{~mm})$, Kommerell's diverticulum. An additional finding was the common origin of the right and left carotid arteries from the aortic arch. The patient's discomfort indicated operative repair of this condition. A vascular clamp was applied to the right subclavian artery at its origin from the aortic arch. This artery was divided and the distal portion was trimmed; then an end-to-side anastomosis was made with the right common carotid artery. Our patient reported complete resolution of his symptoms and tolerated a regular diet without dysphagia.

\section{Discussion}

Dysphagia is defined as difficulty in swallowing food (semisolid or solid), liquid, or both [11]. During normal swallowing, the bolus is propelled from the oral cavity through the pharynx and down the esophagus. Dysphagia occurs when there is a problem with bolus containment and/or propulsion and may occur at the oral, pharyngeal, and/or esophageal phases of swallowing [5, 11-13]. Esophageal dysphagia can arise from a variety of causes such as motility disorders and mechanical and inflammatory diseases [14] shown below.

Causes of esophageal dysphagia in children are as follows

GERD.

Eosinophilic esophagitis.

Tracheoesophageal fistula and esophageal atresia.

Ingestional injuries:

Caustic.

Foreign body.

Congenital diaphragmatic hernia.

Cicatricial stenosis.

Esophageal diverticulae.

Motor disorders:

Achalasia.

Diffuse esophageal spasm.

Nutcracker esophagus.

Hypertensive lower esophageal sphincter.
Extraesophageal compression:

Mediastinal masses (lymphoma, lymph nodes, and thyromegaly).

Vascular compression (dysphagia lusoria, dysphagia aortica, and cardiomegaly).

Systemic diseases:

Crohn's disease.

Leiomyomatosis.

Iatrogenic complications:

Radiotherapy.

Drugs.

Postsurgical complications.

It is rarely caused by extrinsic compression of the esophagus from any vascular anomaly of the aortic arch [15]. ARSA is the most common congenital anomaly of the aortic arch and has a prevalence ranging from $0.5 \%$ to $1.8 \%$ in the general population $[7,16,17]$. Although most cases of this anomaly are asymptomatic, symptoms may appear when a "ring" completely encircles the trachea or the esophagus. Extrinsic compression of the esophagus may lead to dysphagia $[18,19]$. Symptoms, when present, occur at the two extremes of life. In infants, the trachea is compressible; therefore, the typical signs and symptoms of the compression by arteria lusoria are respiratory, such as wheezing, stridor, recurrent pneumonia, and cyanosis. Dysphagia mostly occurs in adults, in whom respiratory symptoms are rare $[8,20]$; adequate management of dysphagia includes a detailed history, evaluation with barium radiography, upper endoscopy, and manometry [14]. The best initial diagnosis would be a barium swallow that will allow for visualization of the esophagus via contrast radiography to determine if there is any evidence of a narrowing due to a stricture, an intraluminal mass, or extraluminal compression [4]. In ARSA, barium esophagogram is often suggestive, showing oblique compression of the esophagus at the level of the third and the fourth thoracic vertebrae [16, 21]. Upper gastrointestinal endoscopy may show prominent aortic pulsation but it is not necessary for the diagnosis. CT or MRI (magnetic resonance imaging) angiography has replaced conventional angiography and is considered the gold standard for the diagnosis. It not only does confirm the diagnosis but also helps to plan the operation and to exclude aneurysm of the aorta or presence of other associated anomalies [17]. Echocardiography has the advantage of a comprehensive assessment of intracardiac anatomy and function. In the presence of respiratory symptoms, the evaluation normally begins with chest radiography [22]. When noisy breathing, stridor, or brassy cough is evident flexible airway endoscopy is the procedure of choice. Despite the accuracy of both MRI and CT in evaluating the nature of the vascular compression of the airways, current techniques do not reliably distinguish between dynamic and static airway narrowing, and coexisting (laryngo) tracheo- or bronchomalacia or tracheal rings can be differentiated with flexible bronchoscopy [23]. Manometry 
cannot be used to diagnose the condition nor has it been of any assistance in distinguishing which patients may benefit from surgery [21]. The treatment depends on the symptoms, age comorbidity, and concomitant vascular abnormalities of each patient [7]. Surgical approach is indicated when ARSA is symptomatic or has evidence of aneurysm [9]. Various surgical approaches can be used, each with its own advantages and limitations [24]. A symptomatic ARSA can be safely repaired through minimally invasive surgery and endovascular techniques, although symptoms do not always regress. Aggressive treatment of an aneurysmal lusorian artery should be proposed, given the rapid natural evolution towards rupture and high mortality of this complication, despite high operative mortality associated with this elective procedure. Endovascular exclusion is an option in patients who are not good surgical candidates [19].

Summarizing, dysphagia is any disturbance in swallowing that may involve oropharyngeal or esophageal phase. Esophageal dysphagia can arise from a variety of disorders and it is rarely caused by arteria lusoria. Symptoms of ARSA are usually different according to age: children predominantly have respiratory symptoms while dysphagia, cough, and chest pain occur mostly in adults. On the contrary, our patient presented with a long history of dysphagia, associated with chest pain and slow feeding. In conclusion, ARSA is a rare cause of dysphagia but it should be taken into account in the differential diagnosis. It is important to be aware of its existence and features to allow an early diagnosis and avoid unnecessary therapeutic interventions. Only an early detection can prevent or minimize complications: unlike adults, children have rapidly developing body systems and even short-term dysphagia can have a detrimental effect on dietary intake. As a result, swallowing difficulties can interrupt physical growth and cognitive development and cause serious long-term sequelae. For all these reasons, it is imperative to accurately identify and appropriately manage dysphagia in pediatric population.

\section{Abbreviation}

ARSA: Aberrant right subclavian artery.

\section{Disclaimer}

All the authors approved the final paper as submitted and agree to be accountable for all aspects of the work.

\section{Conflict of Interests}

The authors declare that there is no conflict of interests regarding the publication of this paper.

\section{Authors' Contribution}

Claudia Barone, Nicolina Stefania Carucci, and Claudio Romano were involved directly in patient care, conducted a literature search of the topic, drafted the initial paper, and approved the final paper as submitted.

\section{References}

[1] B. P. Garg, "Dysphagia in children: an overview," Seminars in Pediatric Neurology, vol. 10, no. 4, pp. 252-254, 2003.

[2] A. N. Khan, K. Said, M. Ahmad, K. Ali, R. Hidayat, and H. Latif, "Endoscopic findings in patients presenting with oesophageal dysphagia," Journal of Ayub Medical College Abbottabad, vol. 26, no. 2, pp. 216-220, 2014.

[3] E. Vaquero-Sosa, L. Francisco-Gonzalez, A. Bodas-Pinedo et al., "Oropharyngeal dysphagia, and underestimated disorder in pediatrics," Revista Espanola de Enfermedades Digestivas, vol. 107, pp. 113-115, 2015.

[4] A. B. Grossman and J. Markowitz, "A 12-year-old boy with progressive dysphagia," The Medscape Journal of Medicine, vol. 10, no. 10, p. 248, 2008.

[5] J. E. Prasse and G. E. Kikano, "An overview of pediatric dysphagia," Clinical Pediatrics (Philadelphia), vol. 48, no. 3, pp. 247-251, 2009.

[6] L. A. Newman, "Optimal care patterns in pediatric patients with dysphagia," Seminars in Speech and Language, vol. 21, no. 4, pp. 281-291, 2000.

[7] M. González-Sánchez, J. L. Pardal-Refoyo, and A. MartínSánchez, "The aberrant right subclavian artery and dysphagia lusoria," Acta Otorrinolaringologica Espanola, vol. 64, no. 3, pp. 244-245, 2013

[8] M. Polguj, Ł. Chrzanowski, J. D. Kasprzak, L. Stefańczyk, M. Topol, and A. Majos, "The aberrant right subclavian artery (arteria lusoria): the morphological and clinical aspects of one of the most important variations-a systematic study of 141 reports," The Scientific World Journal, vol. 2014, Article ID 292734, 6 pages, 2014.

[9] G. De Araújo, J. W. Junqueira Bizzi, J. Muller, and L. T. Cavazzola, “'Dysphagia lusoria'-right subclavian retroesophageal artery causing intermitent esophageal compression and eventual dysphagia-a case report and literature review," International Journal of Surgery Case Reports, vol. 10, pp. 32-34, 2015.

[10] S. Fukuhara, B. Patton, J. Yun, and T. Bernik, "A novel method for the treatment of dysphagia lusoria due to aberrant right subclavian artery," Interactive Cardiovascular and Thoracic Surgery, vol. 16, no. 3, pp. 408-410, 2013.

[11] A. Wieseke, D. Bantz, L. Siktberg, and N. Dillard, "Assessment and early diagnosis of dysphagia," Geriatric Nursing, vol. 29, no. 6, pp. 376-383, 2008.

[12] P. Dodrill, "Feeding problems and oropharyngeal dysphagia in children," Journal of Gastroenterology and Hepatology Research, vol. 3, no. 5, pp. 1055-1060, 2014.

[13] P. Dodrill and M. M. Gosa, "Pediatric dysphagia: physiology, assessment, and management," Annals of Nutrition and Metabolism, vol. 66, no. 5, pp. 24-31, 2015.

[14] A. Lawal and R. Shaker, "Esophageal dysphagia," Physical Medicine and Rehabilitation Clinics of North America, vol. 19, no. 4, pp. 729-745, 2008.

[15] C. Erami, A. Charaf-Eddine, A. Aggarwal, A. L. Rivard, H. W. Giles, and M. J. Nowicki, "Dysphagia lusoria in an infant," The Journal of Pediatrics, vol. 162, no. 6, pp. 1289-1290, 2013.

[16] P. A. Hart and P. S. Kamath, "Dysphagia lusoria," Mayo Clinic Proceedings, vol. 87, no. 3, p. el7, 2012.

[17] V. Abraham, A. Mathew, V. Cherian, S. Chandran, and G. Mathew, "Aberrant subclavian artery: anatomical curiosity or clinical entity," International Journal of Surgery, vol. 7, no. 2, pp. 106-109, 2009. 
[18] R. R. Venugopal, J. P. Kolwalkar, S. P. Krishnajirao, and M. Narayan, "A novel approach for the treatment of dysphagia lusoria," European Journal of Cardio-Thoracic Surgery, vol. 43, no. 2, Article ID ezs498, pp. 434-436, 2013.

[19] P. O. Myers, J. H. D. Fasel, A. Kalangos, and P. Gailloud, "Arteria lusoria: developmental anatomy, clinical, radiological and surgical aspects," Annales de Cardiologie et d'Angeiologie, vol. 59, no. 3, pp. 147-154, 2010.

[20] S. K. Puri, S. Ghuman, P. Narang, A. Sharma, and S. Singh, "CT and MR angiography in dysphagia lusoria in adults," Indian Journal of Radiology and Imaging, vol. 15, no. 4, pp. 497-501, 2005.

[21] A. D. Rogers, M. Nel, E. P. Eloff, and N. G. Naidoo, "Dysphagia lusoria: a case of an aberrant right subclavian artery and a bicarotid trunk," ISRN Surgery, vol. 2011, Article ID 819295, 6 pages, 2011

[22] A. Licari, E. Manca, G. A. Rispoli, S. Mannarino, G. Pelizzo, and G. L. Marseglia, "Congenital vascular rings: a clinical challenge for the pediatrician," Pediatric Pulmonology, vol. 50, no. 5, pp. 511-524, 2015.

[23] O. Sacco, S. Panigada, N. Solari et al., "Vascular malformations," in ERS Handbook, Paediatric Respiratory Medicine, E. Eber and F. Midulla, Eds., pp. 452-460, European Respiratory Society, Sheffield, UK, 2013.

[24] R. Rathnakar, S. Agarwal, V. Datt, and D. Satsangi, "Dysphagia lusoria with atrial septal defect: simultaneous repair through midline," Annals of Pediatric Cardiology, vol. 7, no. 1, pp. 58-60, 2014. 


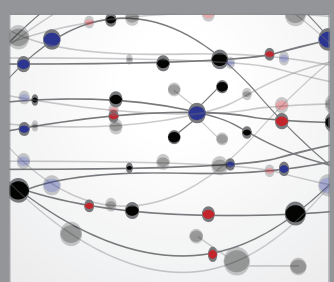

The Scientific World Journal
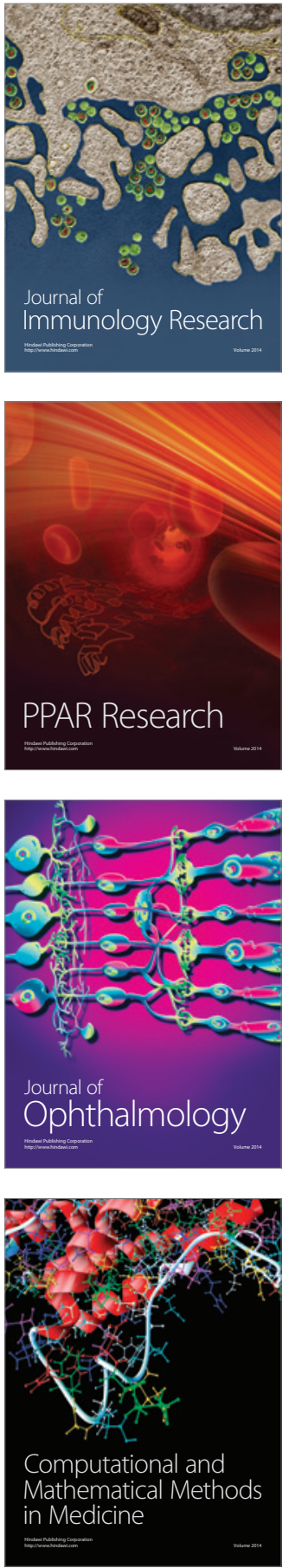

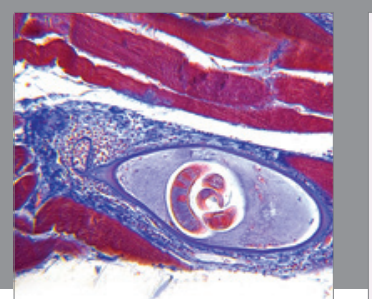

Gastroenterology Research and Practice

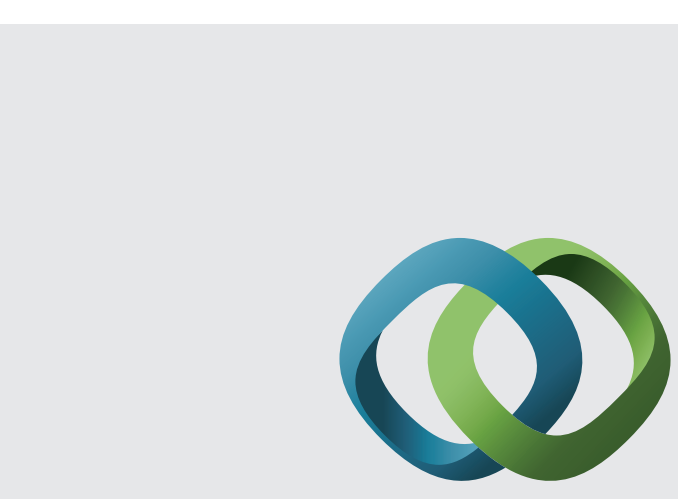

\section{Hindawi}

Submit your manuscripts at

http://www.hindawi.com
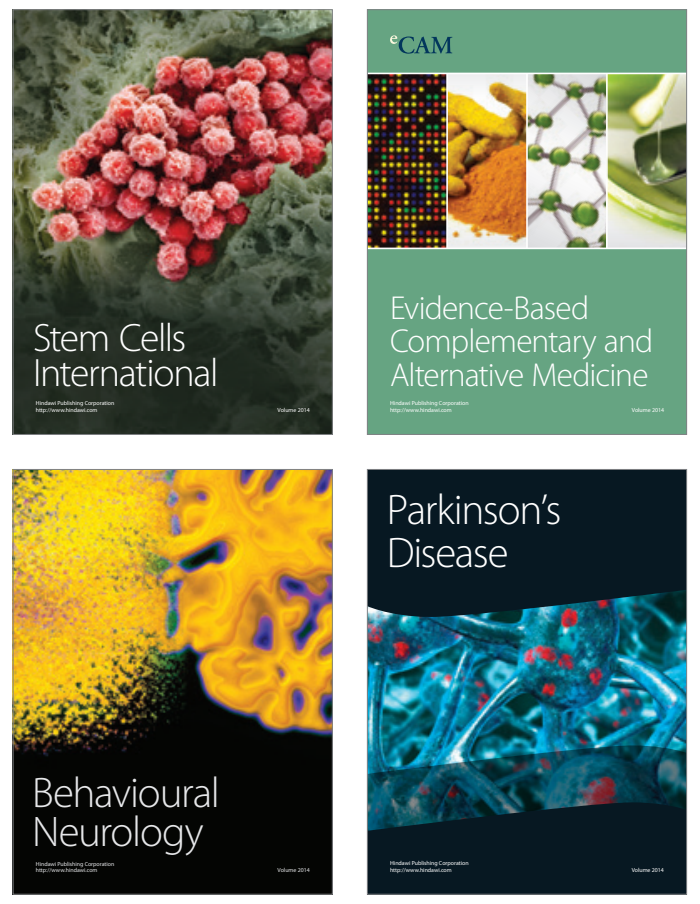
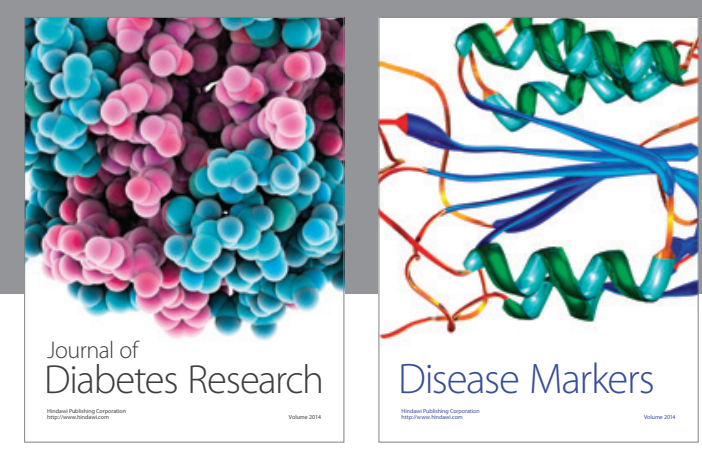

Disease Markers
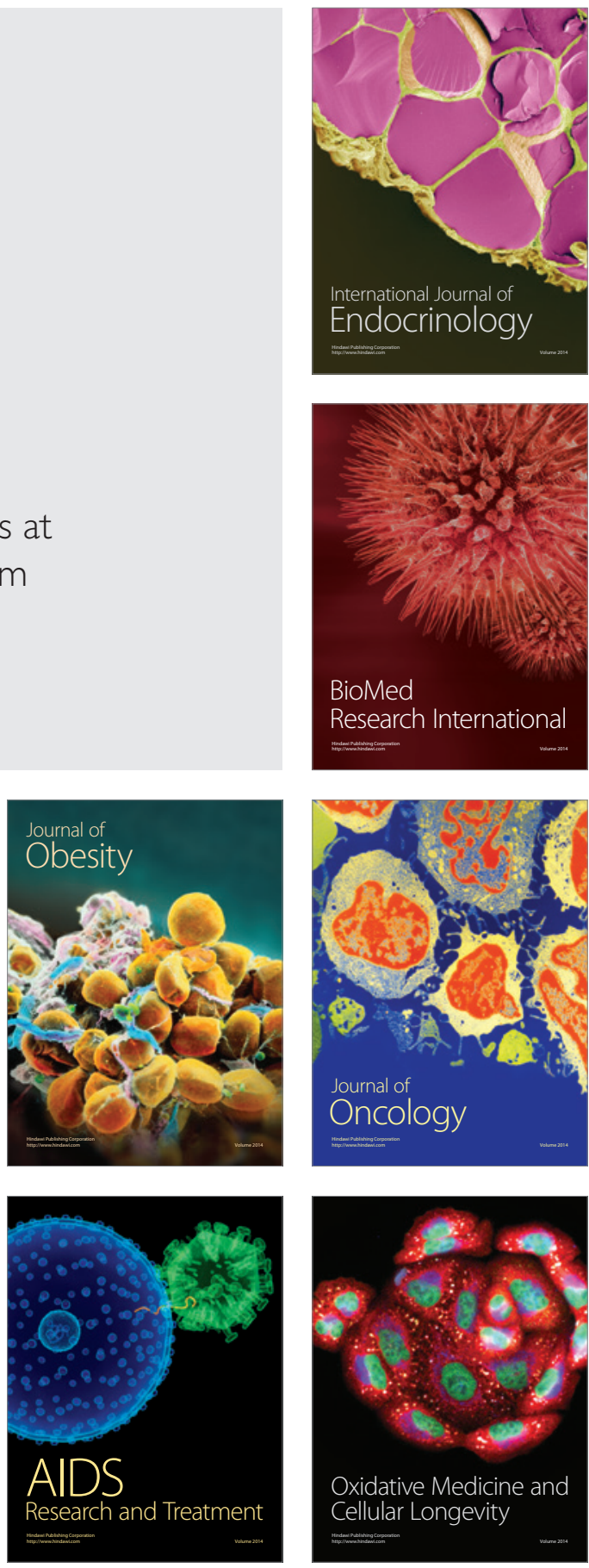